\title{
On the MacWilliams Identity for Classical and Quantum Convolutional Codes
}

\author{
Ching-Yi Lai, Min-Hsiu Hsieh, and Hsiao-feng Lu
}

\begin{abstract}
The weight generating functions associated with convolutional codes (CCs) are based on state space realizations or the weight adjacency matrices (WAMs). The MacWilliams identity for CCs on the WAMs was first established by GluesingLuerssen and Schneider in the case of minimal encoders, and generalized by Forney. We consider this problem in the viewpoint of constraint codes and obtain a simple and direct proof of this MacWilliams identity in the case of minimal encoders. For our purpose, we choose a different representation for the exact weight generating function (EWGF) of a block code, by defining it as a linear combination of orthonormal vectors in Dirac bra-ket notation. This representation provides great flexibility so that general split weight generating functions and their MacWilliams identities can be easily obtained from the MacWilliams identity for EWGFs. As a result, we also obtain the MacWilliams identity for the input-parity weight adjacency matrices of a systematic convolutional code and its dual. Finally, paralleling the development of the classical case, we establish the MacWilliams identity for quantum convolutional codes.
\end{abstract}

\section{INTRODUCTION}

In coding theory, a fundamental theorem is the MacWilliams identity for linear block codes, which provides a precise relation between the weight generating functions of a code and its dual [3]. The weight generating function of a code details the distribution of codeword weights, which can be used to analyze the error performance of the code.

Convolutional codes (CCs) offer a rather different coding paradigm from block codes. The convolutional structure allows a much lower complexity for encoding and decoding circuits without deteriorating its error-correcting ability [4]. The freedistance enumerator is the first notion that counts the weight distribution of the fundamental paths that start and end in the zero states of a code's state diagram without passing any intermediate zero states [5]. This free-distance enumerator is crucial in the error analysis of a CC; however, it was realized later that the MacWilliams identity does not hold for the free distance enumerators [6].

A more refined notion of a weight generating function is the weight adjacency matrix (WAM) [7], [8], [9]. Each entry of this matrix is the weight distribution of all outputs associated with the corresponding state transitions. Unfortunately, a general WAM strongly depends on the underlining encoder

Part of this work was in Proceedings of IEEE Intl. Symp. Inf. Theory 2014 [1], and part of this work was in Proceedings of IEEE Information Theory Workshop 2014 [2].

C.-Y. Lai and M.-H. Hsieh are with the Centre for Quantum Computation \& Intelligent Systems, Faculty of Engineering and Information Technology, University of Technology, Sydney, New South Wales, Australia 2007. M.-H Hsieh is also with the UTS-AMSS Joint Research Laboratory for Quantum Computation and Quantum Information Processing, Academy of Mathematics and Systems Science, Chinese Academy of Sciences, Beijing 100190, China. (emails: cylai0616@gmail.com and Min-Hsiu.Hsieh@uts.edu.au)

H.-F. Lu is with the Department of Electrical and Computer Engineering, at National Chiao Tung University, Taiwan. (email: francis@mail.nctu.edu.tw). and state space description. However, the WAM is shown to be an invariant of a $\mathrm{CC}$ if the encoder is minimal [10]. A breakthrough was made by Gluesing-Luerssen and Schneider in [11], [12], where the MacWilliams identity for the WAMs of a CC and its dual is established. Later, Forney employed the normal graph duality theorem [13] and obtained a more general MacWilliams theorem for various weight generating functions of CCs without the requirement of minimal encoder.

This paper outlines a direct proof of the MacWilliams identity for CCs with minimal encoders. First we define the exact weight generating function (EWGF) of a linear block code as a linear combination of orthonormal vectors in Dirac bra-ket notation. The EWGFs of a code and its dual are directly related by a Fourier transform operator, which gives the MacWilliams identity for linear block codes [3] and facilitates the derivation of MacWilliams identities for general split weight generating functions. As for $\mathrm{CCs}$, we begin with the constraint codes of a CC, introduced by Forney [14], which detail the state evolutions in the state diagram. We show that the dual of a CC with minimal encoder can be defined by the dual of its constraint codes. As a result, we obtain a direct proof of the MacWilliams identity for CCs with minimal encoders, which is simpler than that in [12], without using Forney's normal graph duality theorem. (The assumption of minimal encoders can be removed by using the normal graph duality theorem [14], [13].) In addition, the scalar in the MacWilliams identity for CCs that is missing in [14] is explicitly given in our proof. Moreover, we derive a MacWilliams identity for the input-parity WAMs (IPWAMs) of CCs with systematic encoders, and this answers an open question proposed by Gluesing-Luerssen and Schneider [12]. Finally, we consider weight enumeration over all codewords of a CC and prove its MacWilliams identity. Following that, relations between various weight enumerations, including the free-distance enumerators, are discussed.

Quantum convolutional codes (QCCs) receive great attention for their capabilities in protecting a stream of quantum information in quantum communication, since large blocks of quantum information are very fragile to decoherence [15], [16]. The WAMs and free-distance enumerators of QCCs are defined accordingly and they function like the classical counterparts [17], [18]. We proceed to define the dual code of a QCC within the framework of entanglement-assisted quantum convolutional codes (EA-QCCs) [19], [20]. An EAQCC is defined by a constraint code, which is an EA stabilizer code [21], [22]. The dual code of a stabilizer code is an EA stabilizer code [23] and their MacWilliams identity exist [24], [25], [26]. Our notion of duality follows the normal factor graph duality theorem [13], [14], [27], [28]. Then our classical treatment directly paves the way for the establishment of the MacWilliams identity for EA-QCCs. 
This paper is organized as follows. We introduce the Dirac bra-ket notations and basics of linear block codes and the MacWilliams identity in the next section, so that the materials in this paper are self-complement. In Sec. III, we first discuss classical convolutional codes in the viewpoint of constraints codes and then prove the MacWilliams identities for various notions of weight enumerations of CCs. The MacWilliams identity for EA-QCCs is given in Sec. IV, as well as the definition of the dual code of an EA-QCC. The conclusion follows in Sec. V.

\section{The MacWilliams IDEntity for Split Weight GENERATING FUNCTIONS IN THE DiRAC NOTATION}

We define notations, review the MacWilliams theorem for orthogonal groups, and introduce the Dirac bra-ket notation in this section. This representation allows us to easily derive the MacWilliams identity for general split weight generating functions and facilitates the establishment of our results in the following sections. The readers would have a better understanding of the Dirac notation, which is used in both classical and quantum cases throughout this article.

\section{A. The MacWilliams Identity and the Dirac Notation}

We begin with the Dirac notation, which is used throughout this article. Let $|\phi\rangle$ denote a vector in a complex Hilbert space $\mathcal{H}$, and its adjoint is denoted by $\langle\phi|=| \phi\rangle^{\dagger}$. For $\left|\phi_{1}\right\rangle,\left|\phi_{2}\right\rangle \in$ $\mathcal{H}$, their inner and outer products are denoted by $\left\langle\phi_{1} \mid \phi_{2}\right\rangle$ and $\left|\phi_{1}\right\rangle\left\langle\phi_{2}\right|$, respectively.

Suppose $\mathcal{V}$ is a vector space of dimension $n$ over a finite field $\mathbb{F}_{q}$, where $q=p^{m}$ is a prime power, i.e., $\mathcal{V} \equiv \mathbb{F}_{q}^{n}$. We define a complex Hilbert space $\mathcal{H}_{\mathcal{V}}$, corresponding to $\mathcal{V}$, with an orthonormal basis

$$
\{|\mathbf{v}\rangle: \mathbf{v} \in \mathcal{V}\}
$$

This means that $\left\langle\mathbf{v} \mid \mathbf{v}^{\prime}\right\rangle=\delta_{\mathbf{v}, \mathbf{v}^{\prime}}$, where $\delta_{\mathbf{v}, \mathbf{v}^{\prime}}$ is the Kronecker delta function. Thus $\mathcal{H}_{\mathcal{V}}$ has dimension $q^{n}$. Note that the boldfaced letter $\mathbf{v}$ is used to denote a vector in $\mathcal{V}$, while $|\mathbf{v}\rangle$ is a vector in $\mathcal{H}_{\mathcal{V}}$. We will define weight enumeration of a subset of $\mathcal{V}$ as a linear combination of orthonormal vectors in $\mathcal{H}_{\mathcal{V}}$, rather than a multivariate polynomial commonly used in the literature (see, e.g., Ref. [3]).

Definition 1. The exact weight generating function (EWGF) $g_{\mathcal{C}}^{E}$ of a set $\mathcal{C} \subset \mathcal{V}$ is defined as

$$
g_{\mathcal{C}}^{E}=\sum_{\mathbf{v} \in \mathcal{C}}|\mathbf{v}\rangle \in \mathcal{H}_{\mathcal{V}}
$$

Example 1. An $[n, k, d]_{q}$ linear block code over $\mathbb{F}_{q}$ is a $k$ dimensional subspace of $\mathcal{V}$. Suppose $\mathcal{C}$ is the $[7,3]_{2}$ simplex code with codewords 0000000, 0110011, 1010101, 1100110, 0001111, 0111100, 1011010, and 1101001 [3]. Then $g_{\mathcal{C}}^{E}=$ $|0000000\rangle+|0110011\rangle+|1010101\rangle+|1100110\rangle+|0001111\rangle+$ $|0111100\rangle+|1011010\rangle+|1101001\rangle$.

Let $\langle,\rangle_{\mathcal{V}}:(\mathcal{V}, \mathcal{V}) \rightarrow \mathbb{F}_{p}$ denote an inner product in $\mathcal{V}$. We may sometimes omit the subscript when the underlying vector space is clear from the context. The dual set of $\mathcal{C}$ in $\mathcal{V}$ is

$$
\mathcal{C}^{\perp}=\left\{\mathbf{v}^{\prime} \in \mathcal{V}:\left\langle\mathbf{v}^{\prime}, \mathbf{v}\right\rangle_{\mathcal{V}}=0, \forall \mathbf{v} \in \mathcal{C}\right\}
$$

Let $\mathcal{F}_{\mathcal{V}}$ be a Fourier transform operator defined by

$$
\mathcal{F}_{\mathcal{V}}=\sum_{v^{\prime} \in \mathcal{V}} \sum_{v \in \mathcal{V}} \omega^{\left\langle v^{\prime}, v\right\rangle}\left|v^{\prime}\right\rangle\langle v|
$$

and $\omega=e^{2 \pi i / p}$ is a primitive complex $p$-th root of unity. (Note that $\mathcal{F}_{\mathcal{V}}$ is not normalized: $\mathcal{F}_{\mathcal{V}}^{\dagger} \mathcal{F}_{\mathcal{V}}=|\mathcal{V}| \mathbb{I}$, where $|\mathcal{V}|=$ $q^{n}$ and $\mathbb{I}$ is the identity operator.) The MacWilliams identity connects the weight enumerators of an additively closed set and its dual [3]. It can be rephrased as a Fourier transform in the Dirac notation as in the following theorem. This idea has been used in the construction of quantum Calderbank-ShorSteane (CSS) codes [29], [30], where $g_{\mathcal{C}}^{E}$, up to a normalization factor, is the logical zero state of the CSS code, defined by a classical linear dual-containing code $\mathcal{C}$.

Theorem 1. Suppose $\mathcal{C}$ is an additively closed subset of $\mathcal{V}$ with an EWGF $g_{\mathcal{C}}^{E}=\sum_{v \in \mathcal{C}}|v\rangle$. Then the EWGF of its dual set $\mathcal{C}^{\perp}$ is

$$
g_{\mathcal{C}^{\perp}}^{E}=\frac{1}{|\mathcal{C}|} \mathcal{F}_{\mathcal{V}} g_{\mathcal{C}}^{E}
$$

The proof is straightforward (see [1]). It is natural to generalize this result to the direct product of spaces. Suppose $\mathcal{W}$ is a vector space of dimension $c$ over $\mathbb{F}_{q^{\prime}}$, where $q^{\prime}=p^{m^{\prime}}$. (Note that $m^{\prime}$ may or may not equal to $m$.) We can form a product vector space $\mathcal{V} \times \mathcal{W}$ with an inner product

$$
\left\langle\left(\mathbf{v}_{1}: \mathbf{w}_{1}\right),\left(\mathbf{v}_{2}: \mathbf{w}_{2}\right)\right\rangle_{\mathcal{V} \times \mathcal{W}}=\left\langle\mathbf{v}_{1}, \mathbf{v}_{2}\right\rangle_{\mathcal{V}}+\left\langle\mathbf{w}_{1}, \mathbf{w}_{2}\right\rangle_{\mathcal{W}}
$$

for $\mathbf{v}_{1}, \mathbf{v}_{2} \in \mathcal{V}$ and $\mathbf{w}_{1}, \mathbf{w}_{2} \in \mathcal{W}$, where the addition is in $\mathbb{F}_{p}$. We use the notation $(\mathbf{a}: \mathbf{b})$ to denote the concatenation of two vectors $\mathbf{a}$ and $\mathbf{b}$. Thus we can define a tensor product space $\mathcal{H}_{\mathcal{V} \times \mathcal{W}}=\mathcal{H}_{\mathcal{V}} \otimes \mathcal{H}_{\mathcal{W}}$, which is spanned by

$$
\{|\mathbf{v}\rangle \otimes|\mathbf{w}\rangle: \mathbf{v} \in \mathcal{V}, \mathbf{w} \in \mathcal{W}\}
$$

The tensor product $\otimes$ of two matrices $A$ and $B$ is defined as

$$
A \otimes B=\left[\begin{array}{cccc}
A_{1,1} B & A_{1,2} B & \cdots & A_{1, t} B \\
A_{2,1} B & A_{2,2} B & \cdots & A_{2, t} B \\
\vdots & \vdots & \ddots & \vdots \\
A_{s, 1} B & A_{s, 2} B & \cdots & A_{s, t} B
\end{array}\right],
$$

where $A=\left[A_{i, j}\right]$ is of dimensions $s \times t$. Likewise, we can define a Fourier transform operator $\mathcal{F}_{\mathcal{V} \times \mathcal{W}}$ on $\mathcal{H}_{\mathcal{V}} \otimes \mathcal{H}_{\mathcal{W}}$ :

$$
\mathcal{F}_{\mathcal{V} \times \mathcal{W}}=\sum_{\substack{\left(\mathbf{v}^{\prime}: \mathbf{w}^{\prime}\right) \in \mathcal{V} \times \mathcal{W} \\(\mathbf{v}: \mathbf{w}) \in \mathcal{V} \times \mathcal{W}}} \omega^{\left\langle\left(\mathbf{v}^{\prime}: \mathbf{w}^{\prime}\right),(\mathbf{v}: \mathbf{w})\right\rangle}\left|\mathbf{v}^{\prime}\right\rangle \otimes\left|\mathbf{w}^{\prime}\right\rangle\langle\mathbf{v}| \otimes\langle\mathbf{w}| .
$$

By replacing $\mathcal{V}$ with $\mathcal{V} \times \mathcal{W}$ in Theorem 1 , we can immediately obtain the following corollary.

Corollary 2. Suppose $\mathcal{C}$ is an additively closed subset of $\mathcal{V} \times$ $\mathcal{W}$ with EWGF $g_{\mathcal{C}}^{E}=\sum_{v \in \mathcal{C}}|v\rangle$. Then the EWGF of its dual set $\mathcal{C}^{\perp}$ is

$$
g_{\mathcal{C}^{\perp}}^{E}=\frac{1}{|\mathcal{C}|} \mathcal{F}_{\mathcal{V} \times \mathcal{W}} g_{\mathcal{C}}^{E}
$$

Recently a type of data-and-syndrome error-correcting stabilizer codes are introduced [31], where codes are defined over $\mathbb{F}_{4}^{n} \times \mathbb{F}_{2}^{m}$. Thus Corollary 2 can be applied to these codes.

The Fourier transform operator has a nice property that it 
can be decomposed as a tensor product of Fourier transform operators on its components as in the following lemma.

Lemma 3.

$$
\mathcal{F}_{\mathcal{V} \times \mathcal{W}}=\mathcal{F}_{\mathcal{V}} \otimes \mathcal{F}_{\mathcal{W}}
$$

In particular, $\mathcal{F}_{\mathbb{F}_{q}^{n}}=\mathcal{F}_{\mathbb{F}_{q}}^{\otimes n}$.

\section{B. General Split Weight Generating Functions}

In this subsection we first define the notion of split weight generating function defined by a linear functional. A linear functional that maps $\mathcal{H}_{\mathbb{F}_{q}}$ to the complex numbers $\mathbb{C}$ can be written as

$$
\sum_{\alpha \in \mathbb{F}_{q}} x_{\alpha}\langle\alpha|,
$$

where $x_{\alpha} \in \mathbb{C}$.

Definition 2. A split weight generating function (SWGF) of $\mathcal{C} \subset \mathbb{F}_{q}^{n}$, defined by a linear functional $\gamma: \mathcal{H}_{\mathbb{F}_{q}^{n}} \rightarrow \mathbb{C}$ is

$$
g_{\mathcal{C}}(\gamma)=\left(\bigotimes_{j=1}^{n} \gamma_{j}\right) g_{\mathcal{C}}^{E}
$$

where $\gamma=\bigotimes_{j=1}^{n} \gamma_{j}$ with $\gamma_{j}: \mathcal{H}_{\mathbb{F}_{q}} \rightarrow \mathbb{C}$.

The MacWilliams identity holds for the SWGFs of an additively closed set $\mathcal{C}$ and its dual, and can be easily obtained as an application of Theorem 1 .

Theorem 4. The MacWilliams identity for the SWEs, defined by $\bigotimes_{j} \gamma_{j}$, of an additively closed set $\mathcal{C} \subset \mathbb{F}_{q}^{n}$ and its dual is

$$
g_{\mathcal{C}^{\perp}}\left(\bigotimes_{j} \gamma_{j}\right)=\frac{1}{|\mathcal{C}|} g_{\mathcal{C}}\left(\bigotimes_{j}\left(\gamma_{j} \mathcal{F}_{\mathbb{F}_{q}}\right)\right)
$$

Proof:

$$
\begin{aligned}
& \frac{1}{|\mathcal{C}|} g_{\mathcal{C}}\left(\bigotimes_{j}\left(\gamma_{j} \mathcal{F}_{\mathbb{F}_{q}}\right)\right) \stackrel{(a)}{=} \frac{1}{|\mathcal{C}|} \bigotimes_{j}\left(\gamma_{j} \mathcal{F}_{\mathbb{F}_{q}}\right) g_{\mathcal{C}}^{E} \\
& \stackrel{(b)}{=}\left(\bigotimes_{j} \gamma_{j}\right) \frac{1}{|\mathcal{C}|} \mathcal{F}_{\mathbb{F}_{q}} g_{\mathcal{C}}^{E} \stackrel{(c)}{=}\left(\bigotimes_{j} \gamma_{j}\right) g_{\mathcal{C}^{\perp}}^{E} \stackrel{(d)}{=} g_{\mathcal{C}^{\perp}}\left(\bigotimes_{j} \gamma_{j}\right),
\end{aligned}
$$

where $(a),(d)$ are by Definition $2,(b)$ is from Lemma 3, and (c) follows from Theorem 1

Various notions of weight enumeration can be defined by an appropriate $\gamma$, and their corresponding MacWilliams identities are an direct application of Theorem 4. The Hamming weight of a vector $\mathbf{v} \in \mathbb{F}_{q}^{n}$ is the number of its nonzero components and is denoted by wt $(\mathbf{v})$. Two related weight generating functions are as follows.

Example 2. (Hamming weight generating function (HWGF)) Define a linear functional

$$
\gamma_{\mathbf{H}}(y)=\langle 0|+\sum_{\alpha \in \mathbb{F}_{q} \backslash\{0\}} y\langle\alpha|,
$$

where $y \in \mathbb{C}$. The HWGF of a code $\mathcal{C} \subset \mathbb{F}_{q}^{n}$ is

$$
g_{\mathcal{C}}^{H}(y) \triangleq \sum_{i=0}^{n} A_{i} y^{i}=g_{\mathcal{C}}\left(\gamma_{\mathrm{H}}^{\otimes n}(y)\right),
$$

where $A_{i}$ is the number of codewords in $\mathcal{C}$ of weight $i$. By Theorem 4 we have

$$
g_{\mathcal{C}^{\perp}}^{H}(y)=\frac{(1+(q-1) y)^{n}}{|\mathcal{C}|} g_{\mathcal{C}}^{H}\left(\frac{1-y}{1+(q-1) y}\right) .
$$

Example 3. (Input-parity weight generating function (IPWGF)) Consider an $[n, k]_{q}$ linear block code $\mathcal{C}$ with a systematic generator matrix $G=\left(\begin{array}{ll}I_{k} & A\end{array}\right)$. The first $k$ symbols of a codeword in $\mathcal{C}$ are information symbols, while the remaining $n-k$ symbols are parity symbols. We say a codeword $\mathbf{c}=\left(\mathbf{c}_{I}: \mathbf{c}_{P}\right) \in \mathcal{C}$, where $\mathbf{c}_{I} \in \mathbb{F}_{q}^{k}$ and $\mathbf{c}_{P} \in \mathbb{F}_{q}^{n-k}$, has logical weight $i$, parity weight $r$, and output weight $o$ if wt $\left(\mathbf{c}_{I}\right)=i$, wt $\left(\mathbf{c}_{P}\right)=r$, and wt $(\mathbf{c})=o$. The IPWGF of $\mathcal{C}$ is

$$
g_{\mathcal{C}}^{I P}(x, y) \triangleq \sum_{i=0}^{k} \sum_{j=0}^{n-k} A_{i, j} x^{i} y^{j}=g_{\mathcal{C}}\left(\gamma_{H}^{\otimes k}(x) \otimes \gamma_{H}^{\otimes n-k}(y)\right)
$$

where $A_{i, j}$ is the number of codewords in $\mathcal{C}$ of logical weight $i$ and parity weight $j$.

The $[n, n-k]_{q}$ dual code $\mathcal{C}^{\perp}$ has a systematic generator matrix $H=\left(\begin{array}{ll}-A^{T} & I_{n-k}\end{array}\right)$. In this case, the last $n-k$ symbols of a codeword in $\mathcal{C}^{\perp}$ are information symbols. Thus we have to switch the indeterminates $x$ and $y$ in 12 when applying Theorem 4 After some calculations, we have

$$
\begin{aligned}
g_{\mathcal{C}^{\perp}}^{I P}(x, y)= & \frac{(1+(q-1) y)^{k}(1+(q-1) x)^{n-k}}{|\mathcal{C}|} \\
& \cdot g_{\mathcal{C}}^{I P}\left(\frac{1-y}{1+(q-1) y}, \frac{1-x}{1+(q-1) x}\right) .
\end{aligned}
$$

The input-output weight generating function (IOWGF) of $\mathcal{C}$ can be similarly defined as

$$
g_{\mathcal{C}}^{I O}(x, y) \triangleq \sum_{i=0}^{n} \sum_{j=0}^{k} B_{i, j} x^{i} y^{j}=g_{\mathcal{C}}^{I P}(x y, y),
$$

where $B_{i, j}$ is the number of vectors in $\mathcal{C}$ of output weight $i$ and logical weight $j$. From (13), we have

$$
\begin{aligned}
g_{\mathcal{C}^{\perp}}^{I O}(x, y)= & \frac{(1+(q-1) y)^{k}(1+(q-1) x y)^{n-k}}{|\mathcal{C}|} \\
& \cdot g_{\mathcal{C}}^{I P}\left(\frac{1-y}{1+(q-1) y}, \frac{1-x y}{1+(q-1) x y}\right) .
\end{aligned}
$$

The IPWGF has been introduced in the analysis of error performance [32], [33], with the help of its MacWilliams identity. We will derive similar equations for convolutional codes in the next section, which can be used in error performance analysis.

\section{Convolutional Codes And the MacWilliams IDENTITY}

A. Constraint Code, Weight Adjacency Matrix, and the MacWilliams Identity

Let $\mathcal{C}$ be an $(n, k, m)$ convolutional code over $\mathbb{F}_{q}$ with polynomial generator matrix $G(D) \in \mathbf{M}_{k \times n}\left(\mathbb{F}_{q}[D]\right)$ for some 


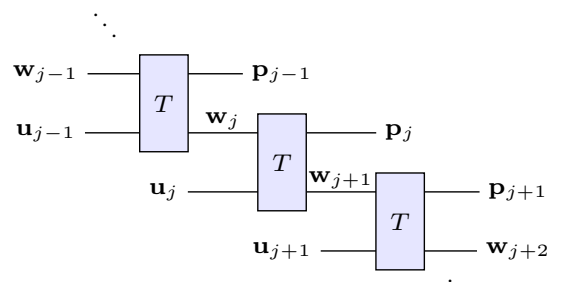

Fig. 1. Circuit diagram of a convolutional encoder with a seed transformation matrix $T$.

indeterminate $D$ and overall constraint length $m$. Then $\mathcal{C}$ is a rank- $k$ submodule of $\left(\mathbb{F}_{q}[D]\right)^{n}$ given by

$$
\mathcal{C}=\left\{\mathbf{u}(D) G(D): \mathbf{u}(D) \in\left(\mathbb{F}_{q}[D]\right)^{k}\right\}
$$

Assume further that $G(D)$ is a minimal encoder of $\mathcal{C}$ with $m$ memory symbols. That is, $G(D)$ is a basic encoder with a right inverse and its overall constraint length $m$ is equal to the maximum degree of its $k \times k$ subdeterminants [4].

As shown in Fig. 1 $G(D)$ outputs $n$ symbols from $k$ logical input symbols at each time step. Let $\mathbf{u}_{j}$ and $\mathbf{p}_{j}$ denote the logical input and physical output symbols at time $j$, respectively, and let $\mathbf{w}_{j}$ and $\mathbf{w}_{j+1}$ denote the $m$ memory symbols before and after encoding at time $j$, respectively. Suppose $(A, B, C, E)$ is a realization of $G(D)$ [34] so that

$$
\begin{aligned}
\mathbf{w}_{j+1} & =\mathbf{w}_{j} A+\mathbf{u}_{j} B \\
\mathbf{p}_{j} & =\mathbf{w}_{j} C+\mathbf{u}_{j} E \\
G(D) & =B\left(D^{-1} I_{m}-A\right)^{-1} C+E
\end{aligned}
$$

where $I_{m}$ is the $m \times m$ identity matrix and $A, B, C, D$ are matrices over $\mathbb{F}_{q}$ of appropriate dimensions. Let

$$
T=\left(\begin{array}{cc}
C & A \\
E & B
\end{array}\right) .
$$

Then (16) and (17) can be written as

$$
\left(\mathbf{p}_{j}: \mathbf{w}_{j+1}\right)=\left(\mathbf{w}_{j}: \mathbf{u}_{j}\right) T .
$$

Thus $T$ is called a seed transformation matrix. We will see later that quantum convolutional codes are similarly defined by a seed transformation unitary operator [17].

Forney introduced the idea of constraint codes [14], which can be used to define the dual code of a CC.

Definition 3. The constraint codes $\mathcal{C}_{(j)}$ of $\mathcal{C}$ is a $[2 m+n, m+$ $k]$ linear block code over $\mathbb{F}_{q}$ given by

$$
\begin{aligned}
\mathcal{C}_{(j)}=\left\{\left(\mathbf{w}_{j}: \mathbf{p}_{j}: \mathbf{w}_{j+1}\right) \in \mathbb{F}_{q}^{n+2 m}:\right. \\
\\
\left.\left(\mathbf{p}_{j}: \mathbf{w}_{j+1}\right)=\left(\mathbf{w}_{j}: \mathbf{u}_{j}\right) T, \text { for } \mathbf{w}_{j} \in \mathbb{F}_{q}^{m}, \mathbf{u}_{j} \in \mathbb{F}_{q}^{k}\right\},
\end{aligned}
$$

where $T$ is a seed transformation matrix of $\mathcal{C}$ defined in (19).

Alternatively, the constraint code $\mathcal{C}_{(j)}$ has a generator matrix

$$
\widetilde{G}=\left(\begin{array}{c|cc}
I_{m} & C & A \\
\hline 0 & E & B
\end{array}\right),
$$

where $I_{m}$ is because the input $\mathbf{w}_{j}$ appears in the codeword, like a systematic encoder; $A, B, C$ and $E$ follow (16) and (17).

In general the constraint codes $\mathcal{C}_{(j)}$ are fixed for all $j$ until termination, where subcodes of $\mathcal{C}_{(j)}$ are used to have all paths converge to the final zero state.
The dual code $\mathcal{C}^{\perp}$ of $\mathcal{C}$ is defined 1 as

$$
\begin{aligned}
\mathcal{C}^{\perp}:= & \left\{\mathbf{c}^{\prime}(D) \in\left(\mathbb{F}_{q}[D]\right)^{n}: \mathbf{c}(D) \mathbf{c}^{\prime}\left(D^{-1}\right)^{T}=0,\right. \\
& \forall \mathbf{c}(D) \in \mathcal{C}\} .
\end{aligned}
$$

The dual code $\mathcal{C}^{\perp}$ can be generated by a minimal encoder with the same overall constraint length $m$ of $\mathcal{C}$ [4], which is important in the following lemma on the constraint codes for the dual of a CC.

Lemma 5. Suppose $\mathcal{C}_{(j)}$ is the constraint code of a minimal encoder $G(D)$. Let

$$
\begin{aligned}
\widehat{\mathcal{C}}_{(j)}=\{ & \left(\mathbf{w}_{j}^{\prime}: \mathbf{p}_{j}^{\prime}: \mathbf{w}_{j+1}^{\prime}\right) \in \mathbb{F}_{q}^{n+2 m}: \mathbf{w}_{j}^{\prime} \mathbf{w}_{j}^{T}+\mathbf{p}_{j}^{\prime} \mathbf{p}_{j}^{T} \\
& \left.-\mathbf{w}_{j+1}^{\prime} \mathbf{w}_{j+1}=0, \forall\left(\mathbf{w}_{j}: \mathbf{p}_{j}: \mathbf{w}_{j+1}\right) \in \mathcal{C}_{(j)}\right\} .
\end{aligned}
$$

Then $\widehat{\mathcal{C}}_{(j)}$ is a $[2 m+n, m+n-k]$ constraint code of the dual code $\mathcal{C}^{\perp}$. In other words, $\widehat{\mathcal{C}}_{(j)}$ has an $(m+n-k) \times(2 m+n)$ generator matrix $\widetilde{H}$ so that $\widetilde{H}^{\prime}=\widetilde{H} \operatorname{diag}\left(I_{m}, I_{n},-I_{m}\right)$ is a parity-check matrix of $\mathcal{C}_{(j)}$.

Proof: Since $G(D)$ is a minimal encoder, the dual encoder has the same overall constraint length as $G(D)$. Suppose $G(D)$ and $H(D)$ are the polynomial generator matrices of $\mathcal{C}$ and $\mathcal{C}^{\perp}$, respectively. Let $\widetilde{H}=\left(\begin{array}{c|cc}I_{m} & C^{\prime} & A^{\prime} \\ \hline 0 & E^{\prime} & B^{\prime}\end{array}\right)$ and $\widetilde{G}$ be as given in (22). As in (18), the polynomial generator matrix of $\mathcal{C}^{\perp}$ is

$$
H(D)=B^{\prime}\left(D^{-1} I_{m}-A^{\prime}\right)^{-1} C^{\prime}+E^{\prime} .
$$

Since $\widetilde{G} \widetilde{H}^{\prime T}=0$, we have

$$
\begin{aligned}
I_{m}+C C^{\prime T}-A A^{\prime T} & =0 \\
E E^{\prime T}-B B^{\prime T} & =0 \\
C E^{\prime T}-A B^{\prime T} & =0 \\
E C^{\prime T}-B A^{\prime T} & =0 .
\end{aligned}
$$

Since a minimal encoder is non-catastrophic and delayfree [4], it is sufficient to prove the duality by showing that $G(D) H\left(D^{-1}\right)^{T}=0$ as follows:

$$
\begin{aligned}
& G(D) H\left(D^{-1}\right)^{T} \\
= & \left(B\left(D^{-1} I_{m}-A\right)^{-1} C+E\right)\left(B^{\prime}\left(D I_{m}-A^{\prime}\right)^{-1} C^{\prime}+E^{\prime}\right)^{T} \\
\stackrel{(a)}{=} & B\left(\left(D^{-1} I_{m}-A\right)^{-1} C C^{\prime T}\left(\left(D I_{m}-A^{\prime}\right)^{-1}\right)^{T}\right. \\
\quad & \left.+\left(D^{-1} I_{m}-A\right)^{-1} A+A^{\prime T}\left(\left(D I_{m}-A^{\prime}\right)^{-1}\right)^{T}+I_{m}\right) B^{\prime T} \\
\stackrel{(b)}{=} & B\left(\left(D^{-1} I_{m}-A\right)^{-1} A A^{\prime T}\left(\left(D I_{m}-A^{\prime}\right)^{-1}\right)^{T}\right. \\
& +\left(D^{-1} I_{m}-A\right)^{-1} A+A^{\prime T}\left(\left(D I_{m}-A^{\prime}\right)^{-1}\right)^{T}+I_{m} \\
& \left.\quad-\left(D^{-1} I_{m}-A\right)^{-1}\left(\left(D I_{m}-A^{\prime}\right)^{-1}\right)^{T}\right) B^{\prime T} \\
\stackrel{(c)}{=} & 0,
\end{aligned}
$$

${ }^{1}$ The duality is defined by $G(D) H(D)^{T}=0$ in [12]. Although not addressed, the duality in [14] is $G(D) H\left(D^{-1}\right)^{\perp}=0$, which is what we adopted in this paper. This explains the additional transpose on the WAM in the formula for MacWilliams identity in [12]. One could also prove the MacWilliams identity from the duality $G(D) H(D)^{\top}=0$ by modifying Lemma 5 appropriately to obtain the identity by Gluesing-Luerssen and Schneider in [11], [12]. However, these points are not addressed in [14] (and perhaps unlikely to be addressed by the method in [14]). 
where $(a)$ follows from (25), (26), and (27); (b) follows from (24); (c) is obtained by sequentially applying the following two equalities:

$$
\begin{aligned}
& I_{m}+\left(D^{-1} I_{m}-A\right)^{-1} A=D^{-1}\left(D^{-1} I_{m}-A\right)^{-1} \\
& I_{m}+A^{\prime T}\left(\left(D I_{m}-A^{\prime}\right)^{-1}\right)^{T}=D\left(\left(D I_{m}-A^{\prime}\right)^{-1}\right)^{T} .
\end{aligned}
$$

Herein, we clarify the notations of $\widehat{\mathcal{C}}_{(j)}$ and $\mathcal{C}_{(j)}^{\perp}$. We denote by $\widehat{\mathcal{C}}_{(j)}$ the constraint code of the dual $\mathrm{CC} \mathcal{C}^{\perp}$, and denote by $\mathcal{C}_{(j)}^{\perp}$ the dual of the constraint code $\mathcal{C}_{(j)}$ of $\mathcal{C}$. Specifically, if $\left(\mathbf{w}_{j}: \mathbf{p}_{j}: \mathbf{w}_{j+1}\right)=\left(\mathbf{w}_{j}: \mathbf{u}_{j}\right) \widetilde{H}$ is a codeword of $\widehat{\mathcal{C}}_{(j)}$ for some $\mathbf{u}_{j} \in \mathbb{F}_{q}^{n-k}$, then $\left(\mathbf{w}_{j}: \mathbf{p}_{j}:-\mathbf{w}_{j+1}\right)$ is a codeword of $\mathcal{C}_{(j)}^{\perp}$. It is straightforward to prove the following lemma.

Lemma 6. The EWGF $g_{\widehat{\mathcal{C}}_{(j)}}^{E}$ of $\widehat{\mathcal{C}}_{(j)}$ is related to the EWGF $g_{\mathcal{C}_{(j)}^{\perp}}^{E}$ of $\mathcal{C}_{(j)}^{\perp}$ by

$$
g_{\hat{\mathcal{C}}_{(j)}}^{E}=\left(I^{\otimes m+n} \otimes \Pi\right) g_{\mathcal{C}_{(j)}^{\perp}}^{E},
$$

where $\Pi=\sum_{\mathbf{w} \in \mathbb{F}_{q}^{m}}|\mathbf{w}\rangle\langle-\mathbf{w}|$ is a permutation on the $m$ memory symbol states and $I^{\otimes m+n}$ is the identity operator on the first $m+n$ symbol states.

Note that in the case that $q$ is a power of $2, \Pi$ is trivial and we have $\widehat{\mathcal{C}}_{(j)}=\mathcal{C}_{(j)}^{\perp}$.

The weight adjacency matrix of $\mathcal{C}$ is the weight enumeration of $\mathcal{C}_{(j)}$ in matrix form.

Definition 4. The weight adjacency matrix (WAM) $\Lambda_{\mathcal{C}_{(j)}}(y)$ of a CC with a constraint code $\mathcal{C}_{(j)}$ is the matrix whose $\left(\mathbf{w}_{j}\right.$, $\mathbf{w}_{j+1}$ ) entry is a HWGF of the output symbols of $\mathcal{C}_{(j)}$ with the memory symbols $\mathbf{w}_{j}$ and $\mathbf{w}_{j+1}$ before and after time $j$, respectively. That is,

$$
\begin{aligned}
\left\langle\mathbf{w}_{j}\left|\Lambda_{\mathcal{C}_{(j)}}(y)\right| \mathbf{w}_{j+1}\right\rangle & \equiv\left(\left\langle\mathbf{w}_{j}\right| \otimes \gamma_{H}^{\otimes n}(y) \otimes\left\langle\mathbf{w}_{j+1}\right|\right) g_{\mathcal{C}_{(j)}}^{E} \\
& =\gamma_{H}^{\otimes n}(y)\left(\sum_{\substack{\mathbf{p}_{j} \in \mathbb{F}_{q}^{n}: \\
\left(\mathbf{w}_{j}: \mathbf{p}_{j}: \mathbf{w}_{j+1}\right) \in \mathcal{C}_{(j)}}}\left|\mathbf{p}_{j}\right\rangle\right) .
\end{aligned}
$$

Now we are ready to derive the MacWilliams identity for convolutional codes [11], [12].

Theorem 7. Suppose the WAM from a minimal encoder of an $(n, k, m)$ CC $\mathcal{C}$ over $\mathbb{F}_{q}$ is $\Lambda_{\mathcal{C}_{(j)}}(x, y)$. Then the WAM for $\widehat{\mathcal{C}}_{(j)}$ is given by

$$
\Lambda_{\widehat{\mathcal{C}}_{(j)}}(y)=\frac{(1+(q-1) y)^{n}}{q^{m+k}} \mathcal{F}_{\mathbb{F}_{q}^{m}} \Lambda_{\mathcal{C}_{(j)}}\left(\frac{1-y}{1+(q-1) y}\right) \mathcal{F}_{\mathbb{F}_{q}^{m}}^{\dagger} .
$$

Proof: From Lemma 6, Theorem 1, and Lemma 3

$$
\begin{aligned}
g_{\hat{\mathcal{C}}_{(j)}}^{E}= & \frac{1}{q^{m+k}} \sum_{\mathbf{w}_{j} \in \mathbb{F}_{q}^{m}} \sum_{\mathbf{w}_{j+1} \in \mathbb{F}_{q}^{m}} \sum_{\substack{\mathbf{p}_{j} \in \mathbb{F}_{q}^{n}: \\
\left(\mathbf{w}_{j}: \mathbf{p}_{j}: \mathbf{w}_{j+1}\right) \in \mathcal{C}_{(j)}}} \\
& \left(\mathcal{F}_{\mathbb{F}_{q}^{m}}\left|\mathbf{w}_{j}\right\rangle\right)\left(\mathcal{F}_{\mathbb{F}_{q}^{n}}\left|\mathbf{p}_{j}\right\rangle\right)\left(\Pi \mathcal{F}_{\mathbb{F}_{q}^{m}}\left|\mathbf{w}_{j+1}\right\rangle\right) .
\end{aligned}
$$

Thus

$$
\begin{aligned}
& \left\langle\mathbf{w}_{j}^{\prime}\left|\Lambda_{\widehat{\mathcal{C}}_{(j)}}(y)\right| \mathbf{w}_{j+1}^{\prime}\right\rangle=\left(\left\langle\mathbf{w}_{j}^{\prime}\right| \otimes \gamma_{H}^{\otimes n}(y) \otimes\left\langle\mathbf{w}_{j+1}^{\prime}\right|\right) g_{\widehat{\mathcal{C}}_{(j)}^{E}}^{E} \\
& =\frac{1}{q^{m+k}} \sum_{\mathbf{w}_{j}} \sum_{\mathbf{w}_{j+1}}\left\langle\mathbf{w}_{j}^{\prime}\left|\mathcal{F}_{\mathbb{F}_{q}^{m}}\right| \mathbf{w}_{j}\right\rangle \\
& \cdot\left(\sum_{\substack{\mathbf{p}_{j} \in \mathbb{F}_{q}^{n}: \\
\left(\mathbf{w}_{j}: \mathbf{p}_{j}: \mathbf{w}_{j+1}\right) \in \mathcal{C}_{(j)}}} \gamma_{H}^{\otimes n}(y) \mathcal{F}_{\mathbb{F}_{q}^{n}}\left|\mathbf{p}_{j}\right\rangle\right)\left\langle\mathbf{w}_{j+1}^{\prime}\left|\Pi \mathcal{F}_{\mathbb{F}_{q}^{m}}\right| \mathbf{w}_{j+1}\right\rangle \\
& \stackrel{(a)}{=} \frac{(1+(q-1) y)^{n}}{q^{m+k}} \sum_{\mathbf{w}_{j}} \sum_{\mathbf{w}_{j+1}}\left\langle\mathbf{w}_{j}^{\prime}\left|\mathcal{F}_{\mathbb{F}_{q}^{m}}\right| \mathbf{w}_{j}\right\rangle \\
& \left\langle\mathbf{w}_{j}\left|\Lambda_{\mathcal{C}_{(j)}}\left(\frac{1-y}{1+(q-1) y}\right)\right| \mathbf{w}_{j+1}\right\rangle\left\langle\mathbf{w}_{j+1}\left|\mathcal{F}_{\mathbb{F}_{q}^{m}}^{\dagger}\right| \mathbf{w}_{j+1}^{\prime}\right\rangle \\
& \stackrel{(b)}{=} \frac{(1+(q-1) y)^{n}}{q^{m+k}}\left\langle\mathbf{w}_{j}^{\prime}\left|\mathcal{F}_{\mathbb{F}_{q}^{m}} \Lambda_{\mathcal{C}_{(j)}}\left(\frac{1-y}{1+(q-1) y}\right) \mathcal{F}_{\mathbb{F}_{q}^{m}}^{\dagger}\right| \mathbf{w}_{j+1}^{\prime}\right\rangle,
\end{aligned}
$$

where $(a)$ follows from the definition of $\Lambda_{\mathcal{C}_{(j)}}(y)$ and $\left\langle\mathbf{w}_{j+1}^{\prime}\left|\Pi \mathcal{F}_{\mathbb{F}_{q}^{m}}\right| \mathbf{w}_{j+1}\right\rangle=\left\langle\mathbf{w}_{j+1}\left|\mathcal{F}_{\mathbb{F}_{q}^{m}}^{\dagger}\right| \mathbf{w}_{j+1}^{\prime}\right\rangle$; (b) follows from $\sum_{\mathbf{w}_{j}}\left|\mathbf{w}_{j}\right\rangle\left\langle\mathbf{w}_{j}\left|=\sum_{\mathbf{w}_{j+1}}\right| \mathbf{w}_{j+1}\right\rangle\left\langle\mathbf{w}_{j+1}\right|=I^{\otimes m}$. The equation $\left\langle\mathbf{w}_{j+1}^{\prime}\left|\Pi \mathcal{F}_{\mathbb{F}_{q}^{m}}\right| \mathbf{w}_{j+1}\right\rangle=\left\langle\mathbf{w}_{j+1}\left|\mathcal{F}_{\mathbb{F}_{q}^{m}}^{\dagger}\right| \mathbf{w}_{j+1}^{\prime}\right\rangle$ can be verified straightforwardly. Then (28) follows directly.

Thus we have provided a more direct and transparent proof than that in [11], [12]. Also, this direct proof gives the scalar in the MacWilliams identity for CCs that is not shown in [14].

Remark: the assumption of minimal encoder in this section could be removed by using the componentwise duality definition of constraint codes and the normal factor graph duality theorem [13], [14]. That is, the normal graph duality theorem implies the definition of dual constraint codes in Lemma 5 without the requirement of minimality. For the rest, we will assume that the dual constraint codes are determined without the encoder being minimal.

In [2], we have this theorem proved using the usual vector notation, instead of the Dirac notation here.

\section{B. The MacWilliams Identity for the Input-Output Weight Adjacency Matrices}

As Gluesing-Luerssen and Schneider noted in [12], the input-output weight generating functions are not invariants of a $\mathrm{CC}$, but rather of the encoder. We will derive the MacWilliams identity for IOWAMs for systematic encoders.

Recall that a seed transformation matrix of an $(n, k, m)$ convolutional code $\mathcal{C}$ is of the form $T=\left(\begin{array}{cc}C & A \\ E & B\end{array}\right)$ and it defines the constraint code with a generator matrix $\widetilde{G}$ given in (22). Here we consider the seed transformation matrix of a systematic encoder, that is, the matrices $C$ and $E$ are in the following specific form: $\left(\begin{array}{c}C \\ E\end{array}\right)=\left(\begin{array}{cc}0 & C_{0} \\ I_{k} & E_{0}\end{array}\right)$, where $C_{0}$ and $E_{0}$ are $m \times(n-k)$ and $k \times(n-k)$ matrices, respectively. We may assume the generator matrix $\widetilde{G}_{S}$ of the constraint code corresponding to a systematic encoder is

$$
\widetilde{G}_{S}=\left(\begin{array}{c|cc|c}
I_{m} & 0 & C_{0} & A_{0} \\
\hline 0 & I_{k} & E_{0} & B_{0}
\end{array}\right) .
$$


Thus $\left(\mathbf{w}_{j}, \mathbf{p}_{j}, \mathbf{w}_{j+1}\right)$ is a codeword of $\mathcal{C}_{(j)}$ for $\mathbf{u}_{j} \in \mathbb{F}_{q}^{k}$ if

$$
\begin{aligned}
\mathbf{w}_{j+1} & =\mathbf{w}_{j} A_{0}+\mathbf{u}_{j} B_{0}, \\
\mathbf{p}_{j} & =\left(\mathbf{u}_{j}: \mathbf{w}_{j} C_{0}+\mathbf{u}_{j} E_{0}\right) \triangleq\left(\mathbf{p}^{I}: \mathbf{p}^{P}\right),
\end{aligned}
$$

where $\mathbf{p}^{I}=\mathbf{u}_{j}$ and $\mathbf{p}^{P}=\mathbf{w}_{j} C_{0}+\mathbf{u}_{j} E_{0}$.

Definition 5. The input-parity weight adjacency matrix (IPWAM) of a systematic convolutional encoder $\widetilde{G}_{S}$ is the matrix $\Lambda_{\mathcal{C}_{(j)}}^{I P}(x, y)$ whose $\left(\mathbf{w}_{j}, \mathbf{w}_{j+1}\right)$ entry is an IPWGF of $\mathcal{C}_{(j)}$ in $x$ and $y$ by $\sum_{i, o} \nu_{i, o} x^{i} y^{o}$, where $\nu_{i, o}$ is the number of $\mathbf{p}_{j}=\left(\mathbf{p}^{I}: \mathbf{p}^{P}\right) \in \mathbb{F}_{q}^{n}$ with wt $\left(\mathbf{p}^{I}\right)=i$ and wt $\left(\mathbf{p}^{P}\right)=o$ so that $\left(\mathbf{w}_{j}: \mathbf{p}^{I}: \mathbf{p}^{P}: \mathbf{w}_{j+1}\right) \in \mathcal{C}_{(j)}$. That is,

$$
=\sum_{\substack{\mathbf{p}_{j} \in \mathbb{F}_{q}^{n}: \\\left(\mathbf{w}_{j}: \mathbf{p}_{j}: \mathbf{w}_{j+1}\right) \in \mathcal{C}_{(j)}}}^{\left\langle\mathbf{w}_{j}\left|\Lambda_{\mathcal{C}_{(j)}}^{I P}(x, y)\right| \mathbf{w}_{j+1}\right\rangle} \gamma_{H}^{\otimes k}(x) \otimes \gamma_{H}^{\otimes n-k}(y)\left|\mathbf{p}_{j}\right\rangle,
$$

where $\gamma_{H}$ is defined in (9).

For simplicity, we assume the corresponding systematic encoder $\widetilde{H}_{S}$ for the constraint code $\widehat{C}_{(j)}$ of $\mathcal{C}^{\perp}$ is of the form $\widetilde{H}_{S}=\left(\begin{array}{c|cc|c}I_{m} & C_{0}^{\prime} & 0 & A_{0}^{\prime} \\ \hline 0 & E_{0}^{\prime} & I_{n-k} & B_{0}^{\prime}\end{array}\right)$. Thus $\left(\mathbf{w}_{j}, \mathbf{p}_{j}, \mathbf{w}_{j+1}\right)$ is a codeword of $\widehat{\mathcal{C}}_{(j)}$ for $\mathbf{u}_{j} \in \mathbb{F}_{q}^{n-k}$ if

$$
\begin{aligned}
\mathbf{w}_{j+1} & =\mathbf{w}_{j} A_{0}^{\prime}+\mathbf{u}_{j} B_{0}^{\prime}, \\
\mathbf{p}_{j} & =\left(\mathbf{w}_{j} C_{0}^{\prime}+\mathbf{u}_{j} E_{0}^{\prime}: \mathbf{u}_{j}\right) \triangleq\left(\mathbf{p}^{P}: \mathbf{p}^{I}\right) .
\end{aligned}
$$

where $\mathbf{p}^{I}=\mathbf{u}_{j}$ and $\mathbf{p}^{P}=\mathbf{w}_{j} C_{0}^{\prime}+\mathbf{u}_{j} E_{0}^{\prime}$.

Similarly to the previous development, it is straightforward to have the following MacWilliams identity for the IPWAMs of a systematic encoder of $\mathcal{C}$ and its dual.

Theorem 8. Suppose the IPWAM of a systematic minimal encoder of an $(n, k, m) \mathrm{CC} \mathcal{C}$ over $\mathbb{F}_{q}$ is $\Lambda_{\mathcal{C}_{(j)}}^{I P}(x, y)$. Then the IPWAM of its dual encoder of $\mathcal{C}^{\perp}$ is

$$
\begin{aligned}
& \Lambda_{\widehat{\mathcal{C}}_{(j)}^{I P}}(x, y) \\
= & \frac{(1+y)^{k}(1+x)^{n-k}}{2^{m}} \mathcal{F}_{\mathbb{F}_{2}}^{\otimes m} \Lambda_{\mathcal{C}_{(j)}}^{I P}\left(\frac{1-y}{1+y}, \frac{1-x}{1+x}\right) \mathcal{F}_{\mathbb{F}_{2}}^{\otimes m},
\end{aligned}
$$

Remark: Given the systematic encoder $\widetilde{G}_{S}$ of $\mathcal{C}$ in 29, it may be natural to define the corresponding systematic encoder $\widetilde{H}_{S}$ for the constraint code $\widehat{C}_{(j)}$ of $\mathcal{C}^{\perp}$ as

$$
\widetilde{H}_{S}=\left(\begin{array}{c|cc|c}
C_{0}^{T} & E_{0}^{T} & I_{n-k} & 0 \\
\hline A_{0}^{T} & B_{0}^{T} & 0 & -I_{m}
\end{array}\right) .
$$

Then a codeword of $\widehat{C}_{(j)}$ is in the reverse order $\left(\mathbf{w}_{j+1}: \mathbf{p}_{j}\right.$ : $\left.\mathbf{w}_{j}\right)=\left(\mathbf{w}_{j+1}: \mathbf{p}^{P}: \mathbf{p}^{I}: \mathbf{w}_{j}\right)$. The MacWilliams identity for the IPWAMs in the above theorem still holds, except that $\Lambda_{\widehat{\mathcal{C}}_{(j)}}^{I P}(x, y)$ is replaced by its transpose in 8 . However, this identity for CCs would require the duality $G(D) H(D)^{T}=0$, which is inconsistent with our development.

Example 4. Consider the constraint code $\mathcal{C}_{(j)}$ of an $(n=$ $2, k=1, m=2$ ) CC over $\mathbb{F}_{2}$ with the following generator and parity-check matrices in the systematic form:

$$
\tilde{G}=\left(\begin{array}{l|l|l|l}
10 & 0 & 1 & 01 \\
01 & 0 & 0 & 10 \\
00 & 1 & 1 & 10
\end{array}\right) \text { and } \tilde{H}=\left(\begin{array}{l|l|l|l}
10 & 1 & 1 & 00 \\
01 & 1 & 0 & 10 \\
10 & 0 & 0 & 01
\end{array}\right) \text {. }
$$

In the case of binary codes, Lemma 5 says that the dual constraint code $\hat{\mathcal{C}}_{(j)}$ is simply the dual code of $\mathcal{C}_{(j)}$, and is generated by $\tilde{H}$. The Fourier transform operator over $\mathbb{F}_{2}$ is

$$
\mathcal{F}_{\mathbb{F}_{2}}=\left[\begin{array}{cc}
1 & 1 \\
1 & -1
\end{array}\right]=\mathcal{F}_{\mathbb{F}_{2}}^{\dagger}
$$

By Definition 5

$$
\Lambda_{\mathcal{C}_{(j)}^{I P}}^{I P}(x, y)=\left(\begin{array}{cccc}
1 & x y & 0 & 0 \\
0 & 0 & y & x \\
x y & 1 & 0 & 0 \\
0 & 0 & x & y
\end{array}\right)
$$

in the ordered basis $\{|00\rangle,|10\rangle,|01\rangle,|11\rangle\}$. By the MacWilliams identity (30), we have,

$$
\Lambda_{\widehat{\mathcal{C}}_{(j)}}^{I P}(x, y)=\left(\begin{array}{cccc}
1 & 0 & x y & 0 \\
x y & 0 & 1 & 0 \\
0 & y & 0 & x \\
0 & x & 0 & y
\end{array}\right),
$$

which is the same as determined from $\hat{\mathcal{C}}_{(j)}$ by Definition 5 .

\section{Weight Enumeration on the Full Trellis Diagram for Con- volutional Codes}

In this subsection we will focus mainly on the Hamming weight enumeration on the codewords of a CC and consider the weight enumeration in one indeterminate since codeword length is not fixed. We reconsider enumerating walks on the full trellis diagram 2 of $\mathcal{C}$ in a matrix. That is, we define

$$
\Lambda_{\mathcal{C}}(y, D):=\left(I_{q^{m}}-\Lambda_{\mathcal{C}_{(j)}}(y) D\right)^{-1}=\sum_{d \geq 0}\left(\Lambda_{\mathcal{C}_{(j)}}(y)\right)^{d} D^{d}
$$

where the $\left(\mathbf{w}, \mathbf{w}^{\prime}\right)$ entry of the matrix $\left(\Lambda_{\mathcal{C}_{(j)}}(y)\right)^{d}$ is the enumeration of the Hamming weights of length- $d$ walks that begin at state $\mathbf{w}$ at time 0 and end at state $\mathbf{w}^{\prime}$ at time $d$ on the full trellis diagram of $\mathcal{C}$. Similarly, we define the full-trellis weight generating function (FWGF)

$$
\Lambda_{\mathcal{C}^{\perp}}(y, D)=\left(I_{q^{m}}-\Lambda_{\widehat{\mathcal{C}}_{(j)}}(y) D\right)^{-1}
$$

Note that we assume the constraint codes $\mathcal{C}_{(j)}$ (and $\widehat{\mathcal{C}}_{(j)}$ ) are the same for all $j$. Then we have the following identity.

Theorem 9. The FWGFs of a $\mathrm{CC} \mathcal{C}$ and its dual are related by the following MacWilliams identity:

$$
\begin{aligned}
& \Lambda_{\mathcal{C}^{\perp}}(y, D) \\
= & \frac{1}{q^{m}} \mathcal{F}_{\mathbb{F}_{q}^{m}} \Lambda_{\mathcal{C}}\left(\frac{1-y}{1+(q-1) y}, \frac{(1+(q-1) y)^{n}}{q^{k}} D\right) \mathcal{F}_{\mathbb{F}_{q}^{m}}^{\dagger}
\end{aligned}
$$

${ }^{2}$ By the full trellis diagram of $\mathcal{C}$ we mean the trellis diagram of $\mathcal{C}$ with arbitrary beginning and ending states. 
Proof: It follows directly from the definitions of $\Lambda_{\mathcal{C}}(y, D), \Lambda_{\mathcal{C}^{\perp}}(y, D)$ and Theorem 8 that

$$
\begin{aligned}
& \Lambda_{\mathcal{C}^{\perp}}(y, D)=\left(I_{q^{m}}-\Lambda_{\widehat{\mathcal{C}}_{(j)}}(y) D\right)^{-1} \\
& =\left[I_{q^{m}}-\frac{(1+(q-1) y)^{n}}{q^{m+k}} \mathcal{F}_{\mathbb{F}_{q}^{m}} \Lambda_{\mathcal{C}_{(j)}}\left(\frac{1-y}{1+(q-1) y}\right) \mathcal{F}_{\mathbb{F}_{q}^{m}}^{\dagger} D\right]^{-1} \\
& =\frac{1}{q^{m}} \mathcal{F}_{\mathbb{F}_{q}^{m}}\left[I_{q^{m}}-\frac{(1+(q-1) y)^{n}}{q^{k}} \Lambda_{\mathcal{C}_{(j)}}\left(\frac{1-y}{1+(q-1) y}\right) D\right]^{-1} \mathcal{F}_{\mathbb{F}_{q}}^{\dagger} \\
& =\frac{1}{q^{m}} \mathcal{F}_{\mathbb{F}_{q}^{m}} \Lambda_{\mathcal{C}}\left(\frac{1-y}{1+(q-1) y}, \frac{(1+(q-1) y)^{n}}{q^{k}} D\right) \mathcal{F}_{\mathbb{F}_{q}^{m}}^{\dagger} .
\end{aligned}
$$

Finally, we state the duality result for full-trellis IPWGF for a systematic $\mathrm{CC} \mathcal{C}$. For any codeword $\mathbf{c} \in \mathcal{C}$, let the weight function of $\mathbf{c}$ be

$$
f_{D}^{\mathrm{IP}}(\mathbf{c})=x^{\sum_{i=0}^{\operatorname{deg}(\mathbf{c})} \mathrm{wt}\left(\mathbf{c}_{i, I}\right)} y^{\sum_{i=0}^{\operatorname{deg}(\mathbf{c})} \mathrm{wt}\left(\mathbf{c}_{i, P}\right)} D^{\mathrm{deg}(\mathbf{c})},
$$

where $\mathbf{c}_{i}=\left(\mathbf{c}_{i, I}: \mathbf{c}_{i, P}\right)$ is defined as in Example 3. Suppose $\mathcal{C}$ is a systematic $\mathrm{CC}$ and $\mathcal{C}^{\perp}$ is the dual code of $\mathcal{C}$. Let

$$
\Lambda_{\mathcal{C}}^{I P}(x, y, D):=\left(I_{q^{m}}-\Lambda_{\mathcal{C}_{(j)}^{I P}}(x, y) D\right)^{-1}
$$

be the full-trellis input-parity weight adjacency matrix (FIPWAM) for walks on the full trellis diagram of $\mathcal{C}$ defined with respect to the above weight function. Along the same way, we have the following theorem.

Theorem 10. The FIPWAMs of a systematic $\mathrm{CC} \mathcal{C}$ and its dual are related by the following MacWilliams identity:

$$
\begin{aligned}
& \Lambda_{\mathcal{C}^{\perp}}^{I P}(x, y, D)=\frac{1}{q^{m}} \mathcal{F}_{\mathbb{F}_{q}^{m}} \Lambda_{\mathcal{C}}^{I P}\left(\frac{1-y}{1+(q-1) y},\right. \\
& \left.\frac{1-x}{1+(q-1) x}, \frac{(1+(q-1) y)^{k}(1+(q-1) x)^{n-k}}{q^{k}} D\right) \mathcal{F}_{\mathbb{F}_{q}^{m}}^{\dagger}
\end{aligned}
$$

\section{Relation Diagram}

The relation of this weight enumeration and the free distance enumeration of a $\mathrm{CC}$ has been discussed in [35], [10]. Here we discuss further with the introduction of MacWilliams identity.

Let $W_{N}^{I P}(x, y)$ denote the input-parity weight enumerator in $x, y$ for the paths of length $N$ and whose initial and final states are both 0 . The total input-parity weight generating function (TIPWGF) $W_{\mathcal{C}}(x, y, D)$ of a $\mathrm{CC}$ is

$$
W_{\mathcal{C}}^{I P}(x, y, D)=\sum_{N \geq 0} W_{N}^{I P}(x, y) D^{N}
$$

From [35, Theorem 4.1], we have

$$
\begin{aligned}
W_{\mathcal{C}}^{I P}(x, y, D) & =\left\langle\mathbf{0}\left|\Lambda_{\mathcal{C}}^{I P}(x, y, D)\right| \mathbf{0}\right\rangle \\
= & \left\langle\mathbf{0}\left|\left(I_{q^{m}}-\Lambda_{\mathcal{C}_{(j)}}^{I P}(x, y) D\right)^{-1}\right| \mathbf{0}\right\rangle,
\end{aligned}
$$

where $|\mathbf{0}\rangle$ is the zero vector in $\mathbb{F}_{q}^{m}$ and $\langle\mathbf{0}|M| \mathbf{0}\rangle$ represents the $(0,0)$-entry of a matrix $M$.

The input-parity free distance enumerator of a CC $W_{\mathcal{C}_{\text {free }}}^{I P}(x, y, D)$ is similarly defined but it counts the paths which do not enter the zero state except at the beginning and end. Since the contribution of the path from state $(\mathbf{0 , 0})$ to state $(\mathbf{0}, \mathbf{0})$ is unwanted, $\left(\Lambda_{\mathcal{C}_{(j)}}^{I P}(x, y)-|\mathbf{0}\rangle\langle\mathbf{0}|\right)$ is considered instead. From [35, Theorem 3.1] (see also [10]), we have

$$
W_{\mathcal{C}_{\text {free }}^{I P}}(x, y, D)=1-\frac{1}{\left\langle\mathbf{0}\left|\left(I_{q^{m}}-\left(\Lambda_{\mathcal{C}_{(j)}}^{I P}(x, y)-|\mathbf{0}\rangle\langle\mathbf{0}|\right) D\right)^{-1}\right| \mathbf{0}\right\rangle} .
$$

Theorem 11. The TIPWGF $W_{\mathcal{C}}^{I P}(x, y, D)$ and the inputparity free distance enumerator $W_{\mathcal{C}_{\text {free }}}^{I P}(x, y, D)$ can be derived from each other:

$$
W_{\mathcal{C}_{\text {free }}^{I P}}^{I P}(x, y, D)=1-\frac{1+W_{\mathcal{C}}^{I P}(x, y, D) D}{W_{\mathcal{C}}^{I P}(x, y, D)} .
$$

Proof: Rewrite (37) as

$$
\begin{aligned}
& \frac{1}{1-W_{\mathcal{C}_{\text {free }}^{I P}}^{I}(x, y, D)} \\
= & \left\langle\mathbf{0}\left|\left[\left(I_{q^{m}}-\Lambda_{\mathcal{C}_{(j)}^{I P}}^{I P}(x, y) D\right)+|\mathbf{0}\rangle\langle\mathbf{0}| D\right]^{-1}\right| \mathbf{0}\right\rangle,
\end{aligned}
$$

and note that $W_{\mathcal{C}}(x, y, D)=\left\langle\mathbf{0}\left|\left(I_{q^{m}}-\Lambda_{\mathcal{C}_{(j)} P}^{I P}(x, y) D\right)^{-1}\right| \mathbf{0}\right\rangle$. By Woodbury identity for matrix inverse, we have (39), which in turn gives the desired equation by considering the $(\mathbf{0}, \mathbf{0})$ entry of both sides.

We summarize the relations 3 of these weight enumerators in Fig. 2. Shearer and McEliece have observed more than 40 years ago that the MacWilliams identity does not exist between $W_{\mathcal{C}_{\text {free }}^{I P}}^{I P}(y, y, D)$ and $W_{\mathcal{C}_{\text {ffe }}^{\perp P}}^{I}(y, y, D)$ [6]. We have an explanation from Figure 2, This is because there is a one-one correspondence, i.e. a duality, between $\Lambda_{\mathcal{C}}^{I P}(x, y, D)$ and $\Lambda_{\mathcal{C}^{\perp}}^{I P}(x, y, D)$ but not for $W_{\mathcal{C}}^{I P}(x, y, D)$ and $W_{\mathcal{C}^{\perp}}^{I P}(x, y, D)$, since $W_{\mathcal{C}}^{I P}(x, y, D)$ corresponds to only the entry of $\Lambda_{\mathcal{C}}^{I P}(x, y, D)$ associated with $(\mathbf{0}, \mathbf{0})$-entry as shown in (35). We point out that, in [14], Forney considered terminated $\mathrm{CCs}$ and defined a normalized Hamming weight distribution $\bar{g}_{\mathcal{C}}(y)=\lim _{N \rightarrow \infty} \operatorname{Tr} \Lambda^{N}(y)$, which has a MacWilliams identity, since for finite $N$, terminated convolutional codes are linear block codes. He showed, by examples, that the free distance enumerator converges to $\bar{g}_{\mathcal{C}}(y)$ rapidly.

The following corollary can be obtained by combining Theorem 10 and Eq. (35).

Corollary 12. The TIPWGF of the dual code of a CC can be determined from its FIPWAM (32):

$$
\begin{aligned}
& W_{\mathcal{C}^{\perp}}^{I P}(x, y, D)=\langle\mathbf{0}| \frac{1}{q^{m}} \mathcal{F}_{\mathbb{F}_{q}^{m}} \Lambda_{\mathcal{C}}^{I P}\left(\frac{1-y}{1+(q-1) y},\right. \\
& \left.\frac{1-x}{1+(q-1) x}, \frac{(1+(q-1) y)^{k}(1+(q-1) x)^{n-k}}{q^{k}} D\right) \mathcal{F}_{\mathbb{F}_{q}^{m}}^{\dagger}|\mathbf{0}\rangle .
\end{aligned}
$$

These results can be directly generalized to input-output free distance enumerators. Similar to (14), let

$$
W_{\mathcal{C}_{\text {free }}}^{I O}(x, y, D)=W_{\mathcal{C}_{\text {free }}}^{I P}(x y, y, D)
$$

${ }^{3}$ We thank anonymous referees for pointing out some mistakes in our previous results [2 Sec. V]. More precisely, we made a mistake on counting the free distance paths when deriving Theorem 11 we were counting the socalled "molecular paths" while we claimed to count the "atomic paths" [35]. Thus (16)-(18) in [2] Sec. V] and the discussion there are incorrect. 


$$
\begin{aligned}
& {\left[\left(I_{q^{m}}-\Lambda_{\mathcal{C}_{(j)}}^{I P}(x, y) D\right)+|\mathbf{0}\rangle\langle\mathbf{0}| D\right]^{-1}} \\
& =\left(I_{q^{m}}-\Lambda_{\mathcal{C}_{(j)}}^{I P}(x, y) D\right)^{-1}-\frac{D}{1+\left\langle\mathbf{0}\left|\left(I_{q^{m}}-\Lambda_{\mathcal{C}_{(j)}}^{I P}(x, y) D\right)^{-1}\right| \mathbf{0}\right\rangle D}\left(I_{q^{m}}-\Lambda_{\mathcal{C}_{(j)}}^{I P}(x, y) D\right)^{-1}|\mathbf{0}\rangle\langle\mathbf{0}|\left(I_{q^{m}}-\Lambda_{\mathcal{C}_{(j)}}^{I P}(x, y) D\right)^{-1}
\end{aligned}
$$

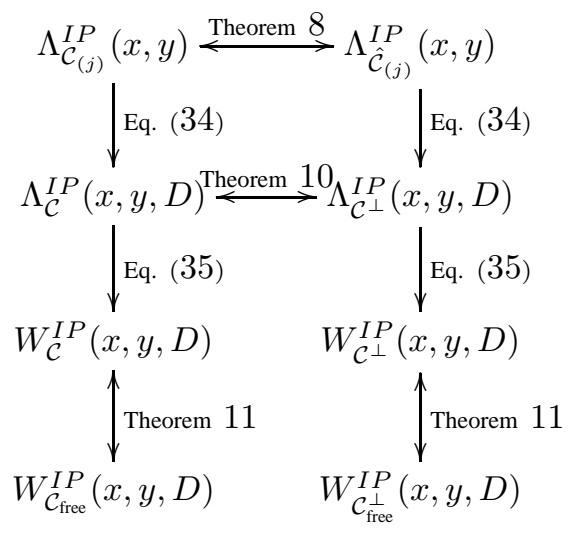

Fig. 2. Relation diagram of various weight enumerations. A relation $A \rightarrow B$ means that $B$ can be derived given $A$. When $x=y$, we have the relations for Hamming weight generating functions.

The bit error rate (BER) $P_{b}$ of a CC over a $q$-ary symmetric channel with error rate $\epsilon$ can be upper bounded by $W_{\mathcal{C}_{\text {free }}^{I O}}^{I O}(x, y, D)$ as follows [9]:

$$
P_{b} \leq\left.\frac{1}{k} \frac{\partial W_{\mathcal{C}_{\text {free }}^{I O}}^{I x}(x, y, D)}{\partial x}\right|_{x=D=1, y=\beta},
$$

where $\beta=2 \sqrt{\epsilon(1-(q-1) \epsilon)}+\epsilon(q-2)$.

\section{Example 4. (Continued.)}

By (40), (36), Theorem 11 and $\Lambda_{\mathcal{C}_{(j)}^{I P}}^{I P}(x, y)$ in 31), we have

$$
\begin{aligned}
W_{\mathcal{C}_{\text {free }}}^{I O}(x, y, D) & =\frac{D^{3} x^{2} y^{5}\left(1+D\left(-1+x^{2}\right) y\right)}{1-D y-D^{2} y-D^{3}\left(-1+x^{2}\right) y^{2}} \\
& =W_{\mathcal{C}_{\text {free }} \perp O}^{I O}(x, y, D),
\end{aligned}
$$

where the MacWilliams identity in Theorem 8 or Theorem 10 is used for the dual code. Thus by 41), the BER of $\mathcal{C}$ or $\mathcal{C}^{\perp}$ over a binary symmetric channel with rate $\epsilon$ is bounded by

$$
P_{b} \leq \frac{2 \beta^{5}\left(1-\beta-\beta^{2}\right)}{(1-2 \beta)^{2}}=2 \beta^{5}+O\left(\beta^{6}\right)
$$

where $\beta=2 \sqrt{\epsilon(1-\epsilon)}$.

The BERs of $\mathcal{C}$ and $\mathcal{C}^{\perp}$ are shown in Fig. 3, together with the analytical upper bound (41). The Viterbi decoder is used with traceback length (TL) 5 and 10. The BERs of the CC and its dual are very close and they are close to the upper bound at low $p$. When TL is greater or equal to 5 , the simulated BER curve is completely below the upper bound. The theoretic upper bound obtained by the input-parity free distance enumerator provides a reasonable performance benchmarking for the CCs.

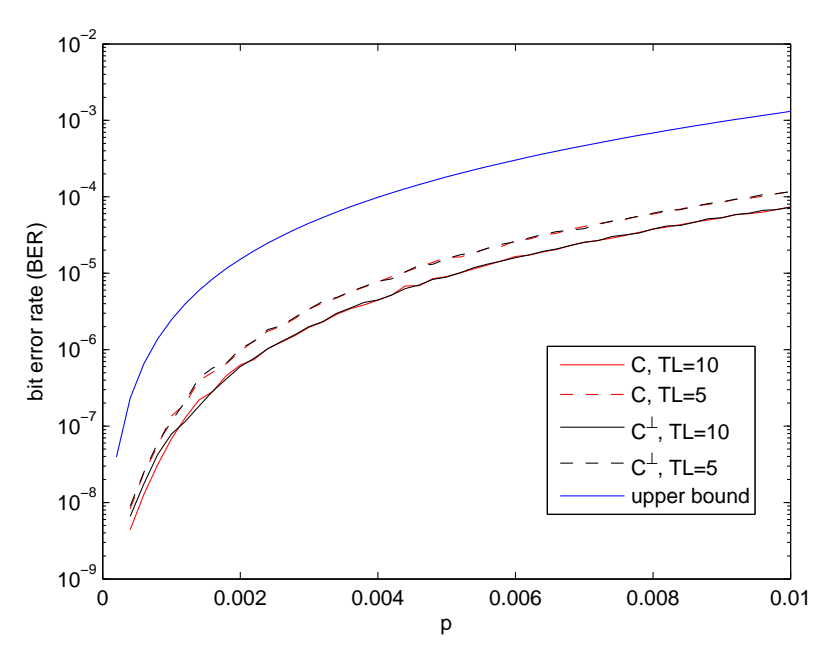

Fig. 3. Comparision of the BER of $\mathcal{C}$ or $\mathcal{C}^{\perp}$ over a binary symmetric channel with rate $p$ and the upper bound 41 . For each $p, 10^{8}$ samples are simulated.

\section{The MacWilliams Identity for QuAntum Convolutional CODES}

\section{A. Pauli Operators and Quantum Codes}

Poulin et al. devised a representation of quantum convolutional codes by a seed transformation and defined the associated state diagram [17], [15]. Entanglement-assisted quantum convolutional codes (EA-QCCs) are developed in [18]. We will use this representation to develop the duality in quantum convolutional codes.

Let us start with some notation. A single-qubit state is a vector in the complex Hilbert space $\mathbb{C}^{2}$ and an $n$-qubit state is a vector in $\mathbb{C}^{2^{n}}$. The Pauli matrices $I=\left[\begin{array}{ll}1 & 0 \\ 0 & 1\end{array}\right]$, $X=\left[\begin{array}{ll}0 & 1 \\ 1 & 0\end{array}\right], Z=\left[\begin{array}{cc}1 & 0 \\ 0 & -1\end{array}\right]$, and $Y=i Z X$ form a basis of the space of linear operators on $\mathbb{C}^{2}$. Let $\mathcal{G}_{1}=$ $\{ \pm I, \pm i I, \pm X, \pm i X, \pm Y, \pm i Y, \pm Z, \pm i Z\}$ be the Pauli group. and let $\mathcal{G}_{n}=\mathcal{G}_{1}^{\otimes n}$ denote the $n$-fold Pauli group. The weight wt $(g)$ of $g \in \mathcal{G}_{n}$ is the number of its components that are not the identity operator. Let $Z_{i}=I^{\otimes i-1} \otimes Z \otimes I^{\otimes n-i}$ and $X_{i}=I^{\otimes i-1} \otimes X \otimes I^{\otimes n-i}$ be the Pauli operators on the $i$-th qubit for convenience and the total number of qubits is clear from the context. For $g, h \in \mathcal{G}_{n}$, the symplectic inner product $\langle,\rangle_{\mathcal{G}_{n}}$ is defined by

$$
\langle g, h\rangle_{\mathcal{G}_{n}}= \begin{cases}0, & \text { if } g h-h g=0 \\ 1, & \text { if } g h+h g=0\end{cases}
$$

A seed transformation $U$ on $\mathbb{C}^{2^{n}}$ is a unitary Clifford 
operator that preserves $\mathcal{G}_{n}$ under conjugation [36], [37], [22]. Suppose $\mathcal{S}$ is an Abelian subgroup of $\mathcal{G}_{n}$ with a set of $n-k$ independent generators defined by a seed transformation $U$

$$
\mathcal{S}=\left\{U\left(I^{\otimes k} \otimes S^{Z}\right) U^{\dagger}: S^{Z} \in\{I, Z\}^{\otimes n-k}\right\}
$$

and $\mathcal{S}$ does not include $-I^{\otimes n}$. An $[[n, k]]$ quantum stabilizer code $\mathcal{C}_{\mathcal{S}}$ is the joint- $(+1)$ eigenspace of $\mathcal{S} \subset \mathcal{G}_{n}$. That is,

$$
\mathcal{C}_{\mathcal{S}}=\left\{|\psi\rangle \in \mathbb{C}^{2^{n}}: g|\psi\rangle=|\psi\rangle, \forall g \in \mathcal{S}\right\} .
$$

$U$ is called a Clifford encoder of the quantum code. In this definition we implicitly assume that the first $k$ qubits before encoding are logical qubits. Let

$$
C(\mathcal{S})=\left\{U\left(L \otimes S^{Z}\right) U^{\dagger}: S^{Z} \in\{I, Z\}^{\otimes n-k}, L \in \mathcal{G}_{k}\right\}
$$

be the centralizer group of $\mathcal{S}$, consisting of operators in $\mathcal{G}_{n}$ that commute with the stabilizers. Thus elements in $C(\mathcal{S})$ are logical operators since they operate on the code space. Note that the orthogonal group of $\mathcal{S}$ is $C(\mathcal{S})$.

Even though an error can be a linear combination of Pauli operators, it will be discretized (in the basis of Pauli operators) after quantum measurements. Also, the overall phase cannot be observed. Thus it suffices to consider errors in

$$
\overline{\mathcal{G}}_{n}=\left\{M_{1} \otimes \cdots \otimes M_{n}: M_{j} \in\{I, X, Y, Z\}\right\}
$$

by ignoring the phase $\pm 1, \pm i$. Note that $\mathcal{S}$ can be chosen to be a subgroup of $\overline{\mathcal{G}}_{n}$. Then the error analysis of the quantum code is equivalent to an classical additive block code in $\mathbb{F}_{2^{2}}$ [36]. Thus the performance of the quantum code is completely characterized by the stabilizer group $\mathcal{S}$.

In the scheme of EA quantum codes, maximally-entangled states $(|00\rangle+|11\rangle) / \sqrt{2}$ are shared between the sender and receiver, which is an +1 eigenstate of $X \otimes X$ and $Z \otimes Z$. (For more details about EA quantum codes, please refer to [21], [22].) Suppose $\mathcal{S} \in \overline{\mathcal{G}}_{n+c}$ is a stabilizer group with $n-k+c$ independent generators

$$
\begin{aligned}
\mathcal{S}= & \left\{U\left(I^{\otimes k} \otimes S^{Z} \otimes S^{E}\right) U^{\dagger} \otimes S^{E}: S^{Z} \in\{I, Z\}^{\otimes n-k-c},\right. \\
& \left.S^{E} \in \overline{\mathcal{G}}_{c}\right\} .
\end{aligned}
$$

Then $\mathcal{S}$ defines an $[[n, k ; c]]$ EA stabilizer code, which is a $2^{k}$-dimensional subspace of $\mathbb{C}^{2^{n}} \otimes \mathbb{C}^{2^{c}}$. Herein, we assume that before encoding, the first $k$ qubits are information qubits, the last $2 c$ qubits are the maximally-entangled states, and the other $n-k-c$ ancillas begin in $|0\rangle$. It is assumed that the qubits held by the receiver before communication are errorfree and thus we neglect operators on those qubits. Then the simplified stabilizer group $\mathcal{S}^{\prime} \subset \overline{\mathcal{G}}_{n}$ is

$$
\begin{aligned}
\mathcal{S}^{\prime}= & \left\{U\left(I^{\otimes k} \otimes S^{Z} \otimes S^{E}\right) U^{\dagger}: S^{Z} \in\{I, Z\}^{\otimes n-k-c},\right. \\
& \left.S^{E} \in \overline{\mathcal{G}}_{c}\right\} .
\end{aligned}
$$

Now $\mathcal{S}^{\prime}$ is no longer Abelian and its centralizer group is $C\left(\mathcal{S}^{\prime}\right)=\left\{U\left(L \otimes S^{Z} \otimes I^{\otimes c}\right) U^{\dagger}: S^{Z} \in\{I, Z\}^{\otimes n-k-c}, L \in \mathcal{G}_{k}\right\}$.

\section{B. Quantum Convolutional Codes}

Like classical CCs, a sequence of seed transformation encoders are applied in the case of quantum convolutional

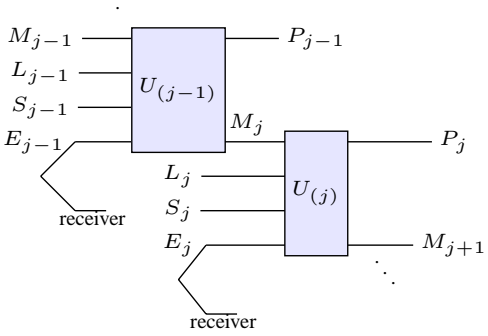

Fig. 4. Circuit diagram of an EA-QCC encoder with a seed transformation $U$

codes as shown in Fig. 4 Note that entanglement (with corresponding operators $E_{j}$ in Fig. (4) is introduced to complete the duality notion of quantum stabilizer codes [23]; that is, the dual code of a stabilizer code is an EA stabilizer code. Please refer to [17], [18] for more details about quantum convolutional codes.

Suppose an $((n, k, c, m))$ EA-QCC $\mathcal{C}$ has $m$ memory qubits, $n$ output qubits, and $k$ logical qubits with the help of $c$ maximally-entangled states at each time step. Suppose $\mathcal{C}$ is defined by seed transformation operator $U_{(j)}$, which is an Clifford encoder on $\mathbb{C}^{2^{n+m}}$. Let

$$
\begin{aligned}
\mathcal{I}^{M} & =\{1, \ldots, m\}, \\
\mathcal{I}^{L} & =\{m+1, \ldots, m+k\}, \\
\mathcal{I}^{A} & =\{m+k+1, \ldots, m+n-c\}, \\
\mathcal{I}^{E} & =\{m+n-c+1, \ldots, m+n\}, \\
\mathcal{I}^{M^{\prime}} & =\{1, \ldots, m\}, \\
\mathcal{I}^{P} & =\{m+1, \ldots, m+n\},
\end{aligned}
$$

which specify the locations of input memory qubits, logical qubits, ancilla qubits, entangled qubits, and output memory qubits, and physical qubits, respectively. The seed transformation $U_{(j)}$ with the input parameters $\left(\mathcal{I}^{M}, \mathcal{I}^{E}, \mathcal{I}^{A}, \mathcal{I}^{L}\right)$ defines the dual constraint code $\mathcal{C}_{(j)}^{\perp}$ as in Definition 3 .

Definition 6. The constraint code $\mathcal{C}_{(j)}$ of $\mathcal{C}$ at time $j$ is an $[[n+$ $2 m, k ; c]]$ EA stabilizer code defined by simplified stabilizer group $\mathcal{S}_{(j)}$ with the following generators:

$$
\begin{array}{ll}
Z_{i}^{M} \otimes g_{i}, X_{i}^{M} \otimes h_{i}, & \text { for } i \in \mathcal{I}^{M} ; \\
I^{M} \otimes g_{i}, I^{M} \otimes h_{i}, & \text { for } i \in \mathcal{I}^{E} ; \\
I^{M} \otimes g_{i}, & \text { for } i \in \mathcal{I}^{A},
\end{array}
$$

where $Z_{i}^{M}, X_{i}^{M}, I^{M} \in \overline{\mathcal{G}}_{m}$ and $g_{i}=U_{(j)} Z_{i} U_{(j)}^{\dagger}, h_{i}=$ $U_{(j)} Z_{i} U_{(j)}^{\dagger} \in \overline{\mathcal{G}}_{n+m}$.

Recall that we consider stabilizer groups in $\overline{\mathcal{G}}_{n}$, which is isomorphic to $\mathbb{F}_{22}^{n}$. Then the normal factor graph duality theorem [14], [13]4 suggests that the dual EA-QCC of $\mathcal{C}$ is defined by the constraint $\operatorname{code} \mathcal{C} \frac{\perp}{(j)}$, which is the $[[n+2 m, c ; k]]$ dual code of $\mathcal{C}_{(j)}$ and has a simplified stabilizer group $\mathcal{S}_{(j)}^{\perp}$

\footnotetext{
${ }^{4}$ Here we employ the normal factor graph duality theorem, which is more general than our development of CCs in Lemma 5
} 
with the following generators:

$$
\begin{aligned}
& Z_{i}^{M} \otimes g_{i}, X_{i}^{M} \otimes h_{i}, \\
& I^{M} \otimes g_{i}, I^{M} \otimes h_{i}, \\
& I^{M} \otimes g_{i},
\end{aligned}
$$$$
\begin{aligned}
& \text { for } i \in \mathcal{I}^{M} \\
& \text { for } i \in \mathcal{I}^{L} \\
& \text { for } i \in \mathcal{I}^{A} \text {. }
\end{aligned}
$$

An EA-QCC and its dual are uniquely defined up to a unitary row operator $R$ that preserves $\mathcal{S}_{(j)}$ and $\mathcal{S}_{(j)}^{\perp}$ [38]. For example, $U_{(j)} R$ is a seed transformation that defines the same EA-QCC as $U_{(j)}$ does if for all $g \in \mathcal{S}_{(j)}$ and $h \in \mathcal{S}_{(j)}^{\perp}$, $R g R^{\dagger} \in \mathcal{S}_{(j)}$ and $R h R^{\dagger} \in \mathcal{S}_{(j)}^{\perp}$, respectively.

Let $M_{(j)}, M_{(j+1)} \in \overline{\mathcal{G}}_{m}$ be the memory operators at time steps $j$ and $j+1$, respectively, and let $\left\{L_{(j)} \in \overline{\mathcal{G}}_{k}\right\}$ be the stream of logical operators. The seed transformation $U_{(j)}$ produces a truncated 5 stabilizer group $\overline{\mathcal{S}}_{(j)}$ and a logical set $C\left(\overline{\mathcal{S}}_{(j)}\right)$ based on $M_{j}$ at each time step $j$. Consequently, the EA-QCC $\mathcal{C}$ is the state space stabilized by the stabilizer group

$$
\bigotimes_{j} \overline{\mathcal{S}}_{(j)}=\left\{\bigotimes_{j} g_{(j)}: g_{(j)} \in \overline{\mathcal{S}}_{(j)}\right\}
$$

More precisely, we have

$$
\begin{aligned}
\overline{\mathcal{S}}_{(j)}= & \left\{P_{(j)} \in\{I, X, Y, Z\}^{\otimes n}: P_{(j)} \otimes M_{(j+1)}\right. \\
& =U_{(j)}\left(M_{(j)} \otimes I^{\otimes k} \otimes S^{Z} \otimes S^{E}\right) U_{(j)}^{\dagger} \text { for } M_{(j)} \in \overline{\mathcal{G}}_{m}, \\
& \left.S^{Z} \in\{I, Z\}^{\otimes n-k-c}, S^{E} \in \overline{\mathcal{G}}_{c}\right\},
\end{aligned}
$$

$$
C\left(\overline{\mathcal{S}}_{(j)}\right)=
$$

$\left\{P_{(j)} \in \mathcal{G}_{n}: P_{(j)} \otimes M_{(j+1)}=U\left(M_{(j)} \otimes L_{(j)} \otimes S^{Z} \otimes I^{\otimes c}\right) U^{\dagger}\right.$

$$
\text { for } \left.M_{(j)} \in\{I, X, Y, Z\}^{\otimes m}, S^{Z} \in\{I, Z\}^{\otimes n-k-c}, L_{(j)} \in \overline{\mathcal{G}}_{k}\right\} \text {. }
$$

Note that an $((n, k, m))$ QCC is a special case of $c=0$.

Remark: One can define a polynomial check matrix $S(D)$ as in [16] and show that $S(D)$ can be obtained from the seed transformation $U$ by finding an equation similar to (18).

The weight generating function of a set $\mathcal{S} \subset \mathcal{G}_{n}$ is

$$
g_{\mathcal{S}}(x, y)=\sum_{w=0}^{n} \nu_{w} x^{n-w} y^{w},
$$

where $\nu_{w}$ is the number of elements in $\mathcal{S}$ of weight $w$. The WAMs of EA-QCCs are defined similarly to the classical case as follows.

Definition 7. The WAM $\Lambda_{\mathcal{C}_{(j)}}(x, y)$ of an EA-QCC $\mathcal{C}$ with constraint code $\mathcal{C}_{(j)}$ defined by a simplified stabilizer group $\mathcal{S}_{(j)}$ is the matrix whose $\left(M_{(j)}, M_{(j+1)}\right)$ entry is the weight generating function of the set of physical output operators $\left\{P_{(j)} \in C\left(\overline{\mathcal{S}}_{(j)}\right)\right\}$ when the input and output memory operators are $M_{(j)}$ and $M_{(j+1)}$, respectively.

Consider $\overline{\mathcal{G}}_{1}=\{I, X, Y, Z\}$. By (3), the matrix representation of the Fourier transform operator $\mathcal{F}_{\overline{\mathcal{G}}_{1}}$ in the ordered basis

\footnotetext{
${ }^{5}$ Note that the part of operators on memory qubits are discarded in this truncated stabilizer group, which is slightly different in the definition of the simplified stabilizer group of an EA stabilizer code.
}

$$
|I\rangle,|X\rangle,|Y\rangle,|Z\rangle \text { is }
$$

$$
\mathcal{F}_{\overline{\mathcal{G}}_{1}}=\left(\begin{array}{cccc}
1 & 1 & 1 & 1 \\
1 & 1 & -1 & -1 \\
1 & -1 & 1 & -1 \\
1 & -1 & -1 & 1
\end{array}\right),
$$

where the symplectic inner product (43) is used. Then the MacWilliams identity for EA-QCCs is as follows.

Theorem 13. Suppose the WAM of an $((n, k, c, m))$ EA-QCC $\mathcal{C}$ is $\Lambda_{\mathcal{C}_{(j)}}(y)$. Then the WAM of its dual $\mathcal{C}^{\perp}$ is

$$
\Lambda_{\mathcal{C}_{(j)}^{\perp}}(y)=\frac{(1+3 y)^{n}}{4^{m} 4^{k} 2^{n-k-c}} \mathcal{F}_{\overline{\mathcal{G}}_{1}}^{\otimes m} \Lambda_{\mathcal{C}_{(j)}}\left(\frac{1-y}{1+3 y}\right) \mathcal{F}_{\overline{\mathcal{G}}_{1}}^{\otimes m} .
$$

Proof: Since the WAM $\Lambda_{\mathcal{C}_{(j)}}(y)$ is counting the weight enumeration of the centralizer group $C\left(\mathcal{S}_{(j)}\right)$ of an $[[n+$ $2 m, k, c]]$ stabilizer code, we have

$$
\left|C\left(\mathcal{S}_{(j)}\right)\right|=2^{2 m} 2^{2 k} 2^{n-k-c} .
$$

By Lemma 3

$$
\mathcal{F}_{\overline{\mathcal{G}}_{m}}=\mathcal{F}_{\overline{\mathcal{G}}_{1}}^{\otimes m} \text {. }
$$

The rest of the proof parallels that of Theorem 7 and is omitted.

The MacWilliams identity holds for the EA-QCCs, catastrophic or noncatastrophic, recursive or nonrecursive (see Refs [17], [18] for these definitions). For examples please refer to [1].

\section{CONCLUSION}

With a different representation of the EWGF in our paper, we provided a direct proof of the MacWilliams identity for the convolutional codes. This method allows us to develop the MacWilliams identity for the IOWAMs of a CC and its dual with systematic encoders, which answered an open question in [12]. The input-output weight distributions are an essential part in the error analysis of iterative decoding, in particular for turbo codes. Our result could potentially lead to preliminary error analysis of both classical and quantum turbo codes. Applications of the IOWAM for turbo codes can be found in [39], for example, where they explicitly demonstrated the usefulness of IOWAMs for turbo codes. By considering expected weight distributions of parallel and serially concatenated ensembles, the error floor under MAP decoding can be predicted by input-output weight enumerators of minimal codewords in the asymptotic case. In addition, one of us has developed an error analysis of linear block codes based on the MacWilliams identity for IOWGF in [32]. A similar error analysis for CCs and turbo codes could be potentially obtained, and is our ongoing investigation.

Mathematically speaking, various forms of weight enumerators can be seen as generalizations of one another, and each comes from a different way of enumerating the underlying abelian group, the $\mathbb{F}_{q}$ vector space. Practically, these weight enumerators have rich applications in analyzing the error performance of a code. To elaborate, the usual Hamming weight enumerator can be used to provide an expression for the "codeword error probability" of a code [3]. On the other 
hand, to get down to the bit error rate (BER), one must require the input-output weight generating function (IOWGF) [39]. Yet, when it comes to the non-binary channel of an $M$-ary modulation, the IOWGF is no longer sufficient for obtaining an expression for BER; it is the complete weight generating function being called for. Perhaps the most extreme case is when the channel is non-binary and time-varying, that is, the channel statistics varies at each transmission; then to deduce a BER expression in such a scenario we must have the exact weight generating function (EWGF). To sum up, the usual Hamming weight enumerator is only applicable to an error analysis for the simplest channel and offers very limited information about the structure of a code; on the contrary, the EWGF, which we used in the paper, is the most general one and is applicable to many other channels.

\section{ACKNOWLEDGMENT}

CYL was supported by the Australian Research Council (ARC) under Grant DP120103776. MH was supported by the UTS Chancellors postdoctoral research fellowship and UTS Early Career Researcher Grants Scheme during the early development of this work. $\mathrm{MH}$ is now supported by an ARC Future Fellowship under Grant FT140100574.

\section{REFERENCES}

[1] C.-Y. Lai and M.-H. Hsieh, "The MacWilliams identity for quantum convolutional codes," in Proc. IEEE Intl. Symp. Inf. Theory, June 2014, pp. 911-915.

[2] C.-Y. Lai, M.-H. Hsieh, and H.-f. Lu, "A complete MacWilliams theorem for convolutional codes," in Proc. 2014 IEEE Inf. Theory Workshop, Nov 2014, pp. 157-161.

[3] F. J. MacWilliams and N. J. A. Sloane, The Theory of Error-Correcting Codes. Amsterdam, The Netherlands: North-Holland, 1977.

[4] J. Forney, G.D., "Convolutional codes I: Algebraic structure," IEEE Trans. Inf. Theory, vol. 16, no. 6, pp. 720-738, Nov 1970.

[5] A. Viterbi, "Convolutional codes and their performance in communication systems," IEEE Trans. Com. Tech., vol. 19, no. 5, pp. 751-772, October 1971.

[6] J. B. Shearer and R. J. McEliece, "There is no MacWilliams identity for convolutional codes," IEEE Trans. Inf. Theory, vol. IT-23, no. 6, pp. 775-776, 1977.

[7] J. Massey and M. Sain, "Codes, automata, and continuous systems: Explicit interconnections," Automatic Control, IEEE Transactions on, vol. 12, no. 6, pp. 644-650, December 1967.

[8] K. Abdel-Ghaffar, "On unit constraint-length convolutional codes," IEEE Trans. Inf. Theory, vol. 38, no. 1, pp. 200-206, Jan 1992.

[9] R. J. McEliece, The Theory of Information and Coding. Cambridge University Press, 2002.

[10] H. Gluesing-Luerssen, "On the weight distribution of convolutional codes," Linear Algebra and its Applications, vol. 408, pp. 298-326, 2005.

[11] H. Gluesing-Luerssen and G. Schneider, "On the MacWilliams identity for convolutional codes," IEEE Trans. Inf. Theory, vol. 54, no. 4, pp. 1536-1550, 2008.

[12] - "A MacWilliams identity for convolutional codes: The general case," IEEE Trans. Inf. Theory, vol. 55, no. 7, pp. 2920-2930, 2009.

[13] J. Forney, G.D., "Codes on graphs: normal realizations," IEEE Trans. Inf. Theory, vol. 47, no. 2, pp. 520-548, 2001.

[14] - "Codes on graphs: Duality and MacWilliams identities," IEEE Trans. Inf. Theory, vol. 57, no. 3, pp. 1382-1397, 2011.

[15] H. Ollivier and J.-P. Tillich, "Description of a quantum convolutional code," Phys. Rev. Lett., vol. 91, p. 177902, Oct 2003. [Online]. Available: http://link.aps.org/doi/10.1103/PhysRevLett.91.177902

[16] G. Forney, M. Grassl, and S. Guha, "Convolutional and tail-biting quantum error-correcting codes," IEEE Trans. Inf. Theory, vol. 53, no. 3, pp. 865-880, 2007.

[17] D. Poulin, J.-P. Tillich, and H. Ollivier, "Quantum serial turbo codes," IEEE Trans. Inf. Theory, vol. 55, no. 6, pp. 2776-2798, 2009.
[18] IEEE Trans. Inf. Theory, vol. 60, no. 2, 2014.

[19] M. M. Wilde and T. A. Brun, "Extra shared entanglement reduces memory demand in quantum convolutional coding," Phys. Rev. A, vol. 79, p. 032313, Mar 2009.

[20] - "Entanglement-assisted quantum convolutional coding," Phys. Rev. A, vol. 81, p. 042333, Apr 2010.

[21] T. A. Brun, I. Devetak, and M.-H. Hsieh, "Correcting quantum errors with entanglement," Science, vol. 314, pp. 436-439, 2006.

[22] T. Brun, I. Devetak, and M.-H. Hsieh, "Catalytic quantum error correction," IEEE Trans. Inf. Theory, vol. 60, no. 6, pp. 3073-3089, June 2014.

[23] C.-Y. Lai, T. A. Brun, and M. M. Wilde, "Duality in entanglementassisted quantum error correction," IEEE Trans. Inf. Theory, vol. 59, no. 6, pp. 4020-4024, 2013.

[24] P. Shor and R. Laflamme, "Quantum analog of the MacWilliams identities for classical coding theory," Phys. Rev. Lett., vol. 78, no. 8, pp. 1600-1602, Feb 1997.

[25] E. M. Rains, "Quantum weight enumerators," IEEE Trans. Inf. Theory, vol. 44, no. 4, pp. $1388-1394,1995$.

[26] A. Ashikhmin and S. Litsyn, "Upper bounds on the size of quantum codes," IEEE Trans. Inf. Theory, vol. 45, no. 4, pp. 1206 - 1215, 1999.

[27] Y. Mao and F. Kschischang, "On factor graphs and the Fourier transform," IEEE Trans. Inf. Theory, vol. 51, no. 5, pp. 1635-1649, 2005.

[28] A. Al-Bashabsheh and Y. Mao, "Normal factor graphs and holographic transformations," IEEE Trans. Inf. Theory, vol. 57, no. 2, pp. 752-763, 2011.

[29] A. R. Calderbank and P. W. Shor, "Good quantum error-correcting codes exist," Phys. Rev. A, vol. 54, no. 2, pp. 1098-1105, 1996.

[30] A. M. Steane, "Multiple particle interference and quantum error correction,” Proc. R. Soc. London A, vol. 452, pp. 2551-2576, 1996.

[31] A. Ashikhmin, C.-Y. Lai, and T. A. Brun, "Correction of data and syndrome errors by stabilizer codes," in Proc. IEEE Int. Symp. Inf. Theory.

[32] H.-F. Lu, P. Kumar, and E. hui Yang, "On the input-output weight enumerators of product accumulate codes," IEEE Communications Letters, vol. 8, no. 8, pp. 520-522, Aug 2004.

[33] M.-C. Chiu and H.-F. Lu, "Accumulate codes based on 1+D convolutional outer codes," Communications, IEEE Transactions on, vol. 57 no. 2, pp. 311-314, February 2009.

[34] J. Forney, G.D., "Structural analysis of convolutional codes via dual codes," IEEE Trans. Inf. Theory, vol. 19, no. 4, pp. 512-518, Jul 1973.

[35] R. J. McEliece, "How to compute weight enumerators for convolutional codes," in Communications and Coding (P. G. Farrell 60th Birthday Celebration), M. Darnell and B. Honory, Eds. New York: Wiley, 1998, p. 121141.

[36] A. R. Calderbank, E. M. Rains, P. W. Shor, and N. J. A. Sloane, "Quantum error correction via codes over GF(4)," IEEE Trans. Inf. Theory, vol. 44, no. 4, pp. 1369-1387, 1998.

[37] D. Gottesman, "Stabilizer codes and quantum error correction," Ph.D. dissertation, California Institute of Technology, Pasadena, CA, 1997. [Online]. Available: http://arxiv.org/abs/quant-ph/9705052

[38] C.-Y. Lai and T. A. Brun, "Entanglement increases the errorcorrecting ability of quantum error-correcting codes," Phys. Rev. A, vol. 88, p. 012320, Jul 2013. [Online]. Available: http://link.aps.org/doi/10.1103/PhysRevA.88.012320

[39] T. Richardson and R. Urbanke, Modern Coding Theory. Cambridge University Press, 2008. 